\title{
Questes
}

vestes Revue pluridisciplinaire d'études médiévales

$7 \mid 2004$

Les tabous

\section{Le tabou : éditorial}

\section{Nelly Labere}

\section{(2) OpenEdition}

\section{Journals}

\section{Édition électronique}

URL : http://journals.openedition.org/questes/2762

DOI : 10.4000/questes.2762

ISSN : 2109-9472

\section{Éditeur}

Les Amis de Questes

\section{Édition imprimée}

Date de publication : 15 juin 2004

Pagination : 1-7

ISSN : 2102-7188

\section{Référence électronique}

Nelly Labere, « Le tabou : éditorial », Questes [En ligne], 7 | 2004, mis en ligne le 15 janvier 2014,

consulté le 23 septembre 2020. URL : http://journals.openedition.org/questes/2762 ; DOI : https:// doi.org/10.4000/questes. 2762 


\section{Le tabou \\ Réflexions sur le sacré et l'impur}

« Ce que personne ne désire faire, on n'a pas besoin de l'interdire »,

Freud, Totem et Tabou, 1912.

\section{Définition usuelle}

Le mot «tabou» semble hanter le discours public. Né dans le terreau d'une ethnologie qui se constitue à la fin du XIX ${ }^{\mathrm{e}}$ siècle, relayé par la psychanalyse, le terme a fini par prendre aujourd'hui un sens proprement politique. « Briser les tabous » signifie, dans le contexte actuel, renverser les limites discursives, reculer les frontières de ce qu'une pression sociale permet ou non de dire, de faire ou de montrer. D'où toutes les références actuelles au tabou dans un contexte culturel (lors du débat sur le roman ou le cinéma pornographique), bioéthique (la question du clonage confronte, par le tabou, les progrès des sciences dures et les limites éthiques et juridiques fixées a priori par la société), social (dans le domaine de l'intimité ou de la différence sexuelle). Imperceptiblement, le tabou a perdu le sens descriptif qui était le sien dans les sciences sociales pour en appeler au subjectif. Face à ce constat, une question s'impose : où fixer les limites du tabou? 
Considéré comme constitutif de la civilisation - quand il s'agit de la prohibition de l'inceste, par exemple - le tabou s'est insensiblement métamorphosé, dans le creuset d'une rhétorique libertaire, en symbole de ce qui fait obstacle à une émancipation sans limites. Alors que Freud désignait le «tabou» comme la manifestation de la civilisation, nous avons tendance aujourd'hui à utiliser ce mot pour renvoyer à ce qui fait obstacle au progrès des techniques et des mœurs. Si l'on aborde la question du tabou d'un point de vue épistémologique, on remarque que, dans les sciences sociales et humaines, l'itinéraire de la notion a été singulier et révélateur de ce que l'on désignait comme «interdit de la main ». Objet de la curiosité des voyageurs au XVIII ${ }^{\mathrm{e}}$ siècle, puis de celle des ethnologues au $\mathrm{XIX}^{\mathrm{e}}$ siècle, le tabou se métamorphose en concept au $\mathrm{XX}^{\mathrm{e}}$ siècle. Terme anachronique pour le Moyen Age, en quoi peut-il nous aider à nous interroger sur ces altérités médiévales?

\section{Définition ethnographique}

Le mot «tabou » a été emprunté au vocabulaire des langues polynésiennes par le célèbre navigateur Cook lors de son passage aux îles Hawaï en 1769. Parfois prononcé tabu, tapu, kapu, tambu, tafu, le mot désigne une réalité dont il convient de s'écarter. Cook, lors de son troisième voyage en 1778, le définit comme s'appliquant «à toutes choses qu'il est interdit de toucher». Ainsi, les 
voyageurs semblent avoir été frappés par la force, le nombre des interdits, la variété des interdits (du religieux au profane) qu'ils rencontraient et qu'ils étaient contraints de respecter. Car le tabou n'a pas seulement des conséquences pour soi mais aussi pour les autres. Il fonctionne comme une règle ou une norme implicite qu'il convient de ne pas transgresser sous peine de remettre en question la structure de la société. Une étude sur les coutumes polynésiennes a montré l'étroite relation entre le système des interdits et la manifestation du pouvoir. La hiérarchie des statuts et des rangs repose sur une échelle de tabous, qui va du moins fort au plus sacré et correspond à des peines expiatoires. Si le tabou fonctionne sur un plan législatif, joue-t-il comme loi morale ou comme prescription politique ? Le tabou est-il de l'ordre du singulier ou du collectif?

C'est James Frazer, l'auteur du Rameau d'or qui a tenté, le premier, de dresser un inventaire des tabous. Il distingue les actes tabous (relations sexuelles avec des étrangers, les interdits portant sur la nourriture et la boisson, le voile du visage, la maison désertée, etc.), les tabous frappant les personnes (les femmes pendant leurs règles et l'enfantement, les guerriers, les assassins, etc.) et les choses taboues (le fer, les armes tranchantes, le sang, la tête, les cheveux et leur coupe, les crachats, les nœuds et les anneaux). Comme la plupart des ethnographes l'ont montré, le tabou s'insère dans un système d'explication du monde dont il est un des instruments. Sous leur plume, le mot se transforme en concept. Il désigne la relation ritualisée à des objets sacrés. En ce sens, il 
renvoie pour les ethnologues à une mentalité « primitive » à laquelle s'oppose la morale et la foi, et trouve en écho dans la dichotomie magie et religion.

\section{Définition psycho-sociologique}

De fait, le tabou crée une distance symbolique. Il suscite alors des mouvements ambivalents de répulsion et d'attirance. C'est dans la complexité de ce sentiment qu'Emile Durkheim a cru déceler, sur le mode archaïque, l'ébauche de la moralité. Freud soulignera, à son tour, dans Totem et Tabou cette ambivalence : «Le tabou est une prohibition très ancienne, imposée du dehors (par une autorité) et dirigée contre les désirs les plus intenses de l'homme. La tendance à la transgresser persiste dans son inconscient ». Dans la perspective ouverte par Freud et Levi-Strauss, le tabou doit être placé au fondement d'une législation qui entrave la communication, la libre circulation et la libre jouissance. On aperçoit dès lors ce qui détermine la fascination qu'il exerce et l'enchantement que provoque le langage des interdits. Le tabou est la condensation d'imparfaites renonciations et de désirs de transgressions.

\section{Pistes de recherche}

C'est la frontière entre public et privé qui devient enjeu de savoir et de pouvoir.

Le tabou en est à la fois sont expression et sa réduction. D'où son expression 
paradoxale : il est une manifestation silencieuse du désir et de la loi, de l'altérité et de la singularité, de l'officieux et de l'officiel. Entre pratique et théorie, le tabou ouvre un vaste champ au questionnement. Mais le tabou, s'il explique le monde, le rend aussi opaque. Il pose la question de la représentation et dans le même temps nie la possibilité de toute représentation. Il affecte enfin le discours comme forme normative menacée, dans son existence, par un objet capable de miner ses fondements. Mais dans le même temps, le discours flirte avec le tabou dans un désir de transgression du langage. Dans le roman médiéval, il est par exemple l'objet d'une transgression que la narration vient compenser. Le texte littéraire reconstruit la défaillance de l'organisation du monde.

Le tabou est donc une affaire de sens et d'essence. Il est un enjeu qui affecte la représentation de soi et de l'autre. Plus que transgression, le tabou médiéval serait subversion. Il offrirait la possibilité d'excéder sans pour autant dépasser et remettre en cause. C'est en ce sens que le tabou pourrait être un instrument pour penser

- l'altérité au Moyen Age - qu'il s'agisse des pratiques alimentaires, des différences sexuelles, etc., le tabou permet de montrer ce qui est interdit ici mais permis chez l'autre. L'altérité se présente alors comme une menace mais aussi comme une possible ouverture.

- l'oralité de la parole et de la consommation. Consommation alimentaire mais aussi consommation sexuelle car le tabou a littéralement partie liée avec la 
sexualité. Noli me tangere (« ne porte pas la main sur moi ») devient l'emblème de l'interdit qui s'énonce sur le mode négatif mais qui se dit pourtant envers et contre tout.

- la difficulté d'une énonciation libre mais critique. A l'image des chroniqueurs qui s'efforcent de relater avec fidélité les événements par une écriture de personnelle, il semble difficile au Moyen Age de dire la vérité sur le prince et son gouvernement. D'où, pour la sottie, et sa critique des particuliers, le recours au dire et au non-dire. Se pose alors la question de la fonction et du type de discours que le sujet applique à son objet.

- la représentation du tabou. Si le verbe ne peut dire, l'image joue-t-elle avec ou contre la parole?

\section{Le tabou : la menace de l'usage idéologique}

Aujourd'hui, le paradoxe du tabou peut être justement d'habiller le « politiquent correct» d'un discours de style libertaire. Au nom de la liberté, on attaque l'institution en s'attachant à exhiber ses limitations. Le tabou devient, de fait, un miroir mais aussi un possible masque. Et l'on peut aller jusqu'à voir dans les études sur le tabou l'indice d'une régression mettant en tension relativisme et moralisme. 
Car parler de tabou n'est pas sans poser le problème de l'utilisation d'un terme moderne et connoté par l'usage des sciences. Le mot oscille entre la norme et l'interdit. Face à cette frontière poreuse, il est parfois difficile de trancher pour savoir s'il s'agit d'une défense ou d'une prohibition.

S'interroger sur les tabous conduit encore à «faire de l'idéologie» en revendiquant quelque chose de son propre discours. En ce sens, le tabou serait presque un terme métalinguistique. Emanant de la société, l'énonciation de tabous ne permettrait pas un dépassement du tabou mais un déplacement du tabou. On en arrive alors à créer un mythe de la destruction du tabou par le langage ou l'image, c'est-à-dire la représentation, sans pour autant considérer que c'est aussi cette représentation qui construit le tabou.

Face à ces problèmes, le médiéviste ne peut que rester prudent dans l'emploi de ce concept anachronique mais néanmoins stimulant. C'est en cherchant dans son propre champ les limites de son utilisation qu'il peut mesurer la complexité de son objet qui témoigne d'une altérité sans cesse à questionner.

Nelly LABERE 